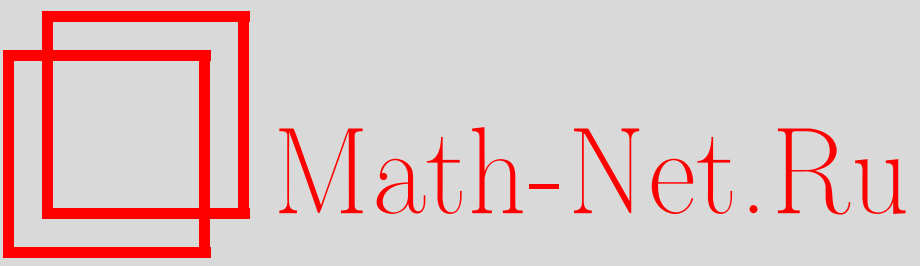

Д. В. Миллионщиков, Когомологии нильмногообразий и теорема Гончаровой, УМH, 2001, том 56, выпуск 4, 153-154

DOI: https://doi.org/10.4213/rm429

Использование Общероссийского математического портала Math-Net.Ru подразумевает, что вы прочитали и согласны с пользовательским соглашением

http://www.mathnet.ru/rus/agreement

Параметры загрузки:

IP : 35.173 .219 .149

26 апреля 2023 г., 13:43:07 


\title{
КОГОМОЛОГИИ НИЛЬМНОГООБРАЗИЙ И ТЕОРЕМА ГОНЧАРОВОЙ
}

\author{
Д. В. Миллионшиков
}

Для построения контрпримера к гипотезе Лаптона-Опри (всякое односвязное симплектическое многообразие является формальным в смысле Сулливана [1]) И.К. Бабенко и И. А. Тайманов рассмотрели в [2] одно очень интересное семейство нильмногообразий $M_{n}$. Впервые это семейство появилось в [3] в связи с изучением алгебр Ландвебера-Новикова.

Определим нилпотентную алгебру Ли $\mathscr{V}_{n}$ базисом $e_{1}, e_{2}, \ldots, e_{n}$ и коммутационными соотношениями

$$
\left[e_{i}, e_{j}\right]= \begin{cases}(j-i) e_{i+j}, & i+j \leqslant n \\ 0, & i+j>n .\end{cases}
$$

По теореме А. И. Мальцева [4] у соответствующей ей односвязной нильпотентной группы Ли $G_{n}$ существует равномерная решетка $\Gamma_{n}$. Нилшмногообразие $G_{n} / \Gamma_{n}$ обозначим как $M_{n}$. В работах В. М. Бухштабера [3], [5] группа $G_{n}$ и ее равномерные решетки красиво определяются при помощи полиномиальных преобразований вещественной прямой.

Когомологии $H^{*}\left(M_{n}, \mathbb{R}\right)$ изоморфны когомологиям $H^{*}\left(\mathscr{V}_{n}\right)$ согласно теореме Номидзу [6]. Важным наблюдением в [2] было

УТВЕРЖДЕНИЕ 1. $M_{2 k}$ является симплектическим многообразием с левочнвариантной симплектической формой

$\Omega_{2 k+1}=\sum_{i+j=2 k+1, i<j}(j-i) \omega_{i} \wedge \omega_{j}=(2 k-1) \omega_{1} \wedge \omega_{2 k}+(2 k-3) \omega_{2} \wedge \omega_{2 k-1}+\cdots+\omega_{k} \wedge \omega_{k+1}$, где $\omega_{1}, \ldots, \omega_{2 k}-$ двойственный $\kappa e_{1}, e_{2}, \ldots, e_{2 k}$ базис левоинвариантных 1-форм.

В результате симплектического раздутия вдоль симплектического вложения $M_{2 k} \rightarrow \mathbb{C} P^{2 k+1}$ получается уже односвязное симплектическое многообразие $\widetilde{M}_{2 k}$, у которого в когомологиях сохраняется нетривиальное тройное произведение Масси, соответствующее $\left\langle\left[\omega_{2}\right],\left[\omega_{1}\right],\left[\omega_{2}\right]\right\rangle$ в $H^{*}\left(\mathscr{V}_{2 k}\right)$. Это и доказывает неформальность $\widetilde{M}_{2 k}$. Вопрос о вычислении $H^{*}\left(M_{n}, \mathbb{R}\right)=$ $H^{*}\left(\mathscr{V}_{n}\right)$ оставался открытым.

Первые же шаги в вычислении $H^{*}\left(\mathscr{V}_{n}\right)$ приводят к известной теореме Гончаровой из теории бесконечномерных алгебр Ли.

Определим алгебру $L_{k}$ как алгебру Ли полиномиальных векторных полей на $\mathbb{R}^{1}$, имеющих $x=0$ нулем кратности $k+1$. Она может быть задана своим базисом

$$
e_{i}=x^{i+1} \frac{d}{d x}, \quad i \in \mathbb{Z}, \quad i \geqslant k ; \quad\left[e_{i}, e_{j}\right]=(j-i) e_{i+j}
$$

Таким образом, алгебра $\mathscr{V}_{n}$ является фактор-алгеброй $\mathscr{V}_{n}=L_{1} / L_{n+1}$.

Используя вторую градуировку $p$ комплекса $C^{*}\left(L_{k}\right)=\oplus_{p} C_{p}^{*}\left(L_{k}\right)$, где $p\left(\omega_{i_{1}} \wedge \cdots \wedge \omega_{i_{q}}\right)=$ $i_{1}+\cdots+i_{q}$ и вычисляя биградуированные когомологии $H_{p}^{q}\left(L_{k}\right)$, Л. В. Гончарова в [7] доказала следующие формулы (гипотеза Гельфанда-Фукса) для чисел Бетти алгебр $L_{k}$ :

$$
\operatorname{dim} H^{q}\left(L_{k}\right)=\left(\begin{array}{c}
q+k-1 \\
k-1
\end{array}\right)+\left(\begin{array}{c}
q+k-2 \\
k-1
\end{array}\right) .
$$

Для вычисления $H^{*}\left(L_{1} / L_{n+1}\right)$ рассмотрим спектральную последовательность Серра-Хoхшильда $\left\{E_{r}^{i, j}, d_{r}\right\}$ пары $\left(L_{1}, L_{n+1}\right)$, сходящуюся к $H^{*}\left(L_{1}\right)$. Полное вычисление всех ее дифференциалов $d_{r}$ приводит к значительным техническим трудностям комбинаторного плана, но можно отметить следующие факты.

Работа выполнена при поддержке Российского фонда фундаментальных исследований (грант № 99-01-00090). 
ЛЕмма 2. При $q \leqslant \frac{1}{3}(n+1)$ в виполняется

$$
\begin{gathered}
E_{3}^{i, j}=E_{\infty}^{i, j}=0, \quad i+j=q, \quad j \neq 0 ; \quad E_{3}^{q, 0}=E_{\infty}^{q, 0}=H^{q}\left(L_{1}\right) ; \\
E_{2}^{q, 0}=H^{q}\left(L_{1} / L_{n+1}\right)=\operatorname{Im} d_{2}\left(E_{2}^{q-2,1}\right) \oplus H^{q}\left(L_{1}\right),
\end{gathered}
$$

$\operatorname{Im} d_{2}\left(E_{2}^{q-2,1}\right)=\left[\Omega_{n+1}\right] H^{q-2}\left(L_{2}\right) \oplus\left[\Omega_{n+1}\right]^{2} H^{q-4}\left(L_{3}\right) \oplus\left[\Omega_{n+1}\right]^{3} H^{q-6}\left(L_{4}\right) \oplus \cdots$,

где $\left[\Omega_{n+1}\right]-$ двумерный класс когомологий формы $\Omega_{n+1}=\sum_{i+j=n+1,1 \leqslant i<j}(j-i) \omega_{i} \wedge$ $\omega_{j}$

СлеДСтвиЕ 3. При $q \leqslant \frac{1}{3}(n+1)$ имеется биградуированный изоморфизм $H^{q}\left(L_{1} / L_{n+1}\right)=H^{q}\left(L_{1}\right) \oplus\left[\Omega_{n+1}\right] H^{q-2}\left(L_{2}\right) \oplus\left[\Omega_{n+1}\right]^{2} H^{q-4}\left(L_{3}\right) \oplus\left[\Omega_{n+1}\right]^{3} H^{q-6}\left(L_{4}\right) \oplus \cdots$

Теперь, используя значения (1) для $\operatorname{dim} H^{q-2 i}\left(L_{i+1}\right)$, мы можем подсчитать размерность $\operatorname{dim} H^{q}\left(L_{1} / L_{n+1}\right)$ при $q \leqslant \frac{1}{3}(n+1)$ :

$$
\operatorname{dim} H^{q}\left(L_{1} / L_{n+1}\right)=\left(\begin{array}{l}
q \\
0
\end{array}\right)+\left(\begin{array}{c}
q-1 \\
0
\end{array}\right)+\left(\begin{array}{c}
q-1 \\
1
\end{array}\right)+\left(\begin{array}{c}
q-2 \\
1
\end{array}\right)+\left(\begin{array}{c}
q-2 \\
2
\end{array}\right)+\left(\begin{array}{c}
q-3 \\
2
\end{array}\right)+\cdots
$$

Последняя сумма есть не что иное, как сумма элементов двух соседних диагоналей треугольника Паскаля, а она, как известно, равняется $(q+2)$-му числу Фиббоначчи $F_{q+2}$. Таким образом, приходим к основной теореме:

ТеОРема 4. Число Бетти $b_{q}\left(M_{n}\right)=\operatorname{dim}\left(H^{q}\left(\mathscr{V}_{n}\right)\right)$ стабилизируется $n p u n \geqslant 3 q-1 u$ равняется $(q+2)$-му числу фиббоначчи $F_{q+2}$ :

$$
b_{1}\left(M_{n}\right)=2, b_{2}\left(M_{n}\right)=3, b_{3}\left(M_{n}\right)=5, \ldots, b_{q}\left(M_{n}\right)=F_{q+2}, \quad n \geqslant 3 q-1 .
$$

Пример 5. Рассмотрим первье размерности $q=1,2,3$ ( $n \geqslant 3 q-1$ в каждом случае):

1) $H^{1}\left(\mathscr{V}_{n}\right)=\left\langle\left[\omega_{1}\right],\left[\omega_{2}\right]\right\rangle$

2) $H^{2}\left(\mathscr{V}_{n}\right)=\left\langle\left[\omega_{1} \wedge \omega_{4}\right],\left[\omega_{2} \wedge \omega_{5}+\frac{3}{2} \omega_{1} \wedge \omega_{6}\right],\left[\Omega_{n+1}\right]\right\rangle$;

3) в $H^{3}\left(\mathscr{V}_{n}\right)$ имеется 5 образующих: $g_{12}, g_{15}$ (образующие из $H^{3}\left(L_{1}\right)$ с второй градуировкой 12, 15 соответственно [7]) и три коцикла

$\left[\omega_{2}\right] \wedge\left[\Omega_{n+1}\right], \quad\left[\omega_{2} \wedge \Omega_{n+2}-n \omega_{3} \wedge \Omega_{n+1}\right], \quad\left[\omega_{2} \wedge \Omega_{n+3}-(n+1) \omega_{3} \wedge \Omega_{n+2}+\frac{n(n+1)}{2} \omega_{4} \wedge \Omega_{n+1}\right]$

$$
\text { əде } \Omega_{n+2}=\sum_{i+j=n+2,2 \leqslant i<j}(j-i) \omega_{i} \wedge \omega_{j}, \Omega_{n+3}=\sum_{i+j=n+3,3 \leqslant i<j}(j-i) \omega_{i} \wedge \omega_{j} .
$$

Автор выражает благодарность Л. А. Алания, И. К. Бабенко, В. М. Бухштаберу и В. Ю. Овсиенко за обсуждение резултатов работы.

\section{СПИСОК ЛИТЕРАТУРЫ}

[1] G. Lupton, J. Oprea // J. Pure Appl. Algebra. 1994. V. 91. Р. 193-207. [2] И. К. Бабенко, И. А. Тайманов // УМН. 1998. Т. 53. № 5. С. 225-226. [3] В. М. Бухштабер, А. В. Шокуров // Функц. анализ и его прил. 1978. Т. 12. № 3. С. 1-11. [4] А. И. Мальцев // Изв. АН СССР. Сер. матем. 1949. Т. 3. С. 9-32. [5] В. М. Бухштабер // УМН. 1999. Т. 54. № 4. C. 161. [6] K. Nomizu // Ann. of Math. 1954. V. 59. P. 531-538. [7] Л. В. Гончарова // Функц. анализ и его прил. 1973. Т. 7. № 2. С. 6-14. 